\title{
The Giant Core Hole Effect in Electron Energy Loss Spectroscopy
}

\author{
P. Rez, L.A.J. Garvie* and A.V. Chizmeshya \\ Center for Solid State Science, Arizona State University, Tempe, AZ 8587-1704 \\ *Department of Geology, Arizona State University, Tempe AZ 85287-1404
}

The treatment of core hole effects in electron energy loss spectroscopy (EELS) and X-ray absorption spectroscopy has been a matter of controversy for many years. The standard treatment assumes that the excited electron experiences a potential equivalent to an atom with the core electron missing. Sometimes this potential is calculated explicitly as in the FEFF codes[1], but frequently the excited atom is just replaced with the atom with the next highest atomic number since the core hole effectively increases the nuclear charge by one. Qualitatively the new potential increases the magnitude of peaks at threshold and shifts them to lower energies. Comparison with experiment shows that incorporating a core hole potential is not a universal panacea. In some cases, notably the oxygen $\mathrm{K}$ edge in oxides, ground state projected density of states calculations give better agreement with experiment, while in other cases the core hole calculation exaggerates the magnitude of near threshold features.

The standard core hole prescription can also fail by underestimating the influence of the core hole. A FEFF8 fully self-consistent Green function cluster calculation [1] gives excellent agreement for $\mathrm{BN}$, yet seriously underestimates the first peak in $\mathrm{B}_{2} \mathrm{O}_{3}$ as shown in Fig 1. A similar effect can be see for the $\mathrm{Be} \mathrm{K}$ edge in $\mathrm{BeO}$ which also shows a sharp peak at threshold. The explanation for this enhancement of the threshold peak can be found in sophisticated theories that use the Bethe Salpeter equation to describe the evolution of the two particle Greens function representing the core hole and the excited electron $[2,3]$. A simple description relies on the analogy with the Fano resonance $[3,4]$, an interference between a bound and continuum state of the same character. Fano resonances greatly enhance the intensity of the $\mathrm{M}_{23}$ edges in the $1^{\text {st }}$ row transition elements and the $\mathrm{N}_{45}$ edges of the rare earths. In this particular case the interference is between a $\mathrm{p}$ state bound by the core hole and continuum states in the low lying part of the conduction band.

A code for Fano resonances was used to calculate the variation of the generalized oscillator strength with energy. This was convoluted with a Gaussian $1 \mathrm{eV}$ wide to take account of instrumental and life-time broadening and then substituted for the corresponding quantity in the FEFF8 output. The results showing enhancement of the first peak are given as Fig 2 for $\mathrm{BeO}$ and Fig 3 for $\mathrm{B}_{2} \mathrm{O}_{3}$. Analysis of the theory shows that the magnitude of the resonance is related to an exchange integral that is solely dependent on atomic properties, and calculations indicate that the effect is greatest for $\mathrm{Be}$ and Bo and less significant for $\mathrm{Li}$ and $\mathrm{Mg}$.

\section{References}

[1] A.L. Ankudinov, B. Ravel, J.J. Rehr and S.D. Conradson, Phys. Rev. B 58 (1998) 7565.

[2] E. L. Shirley, Phys. Rev. Lett. 80 (1998) 794.

[3] G. Strinati, Phys. Rev. Lett. 49 (1982) 1519.

[4] U. Fano, Phys. Rev. 124 (1961) 1866

[5] This work was supported by NSF grant DMR 99-72670 


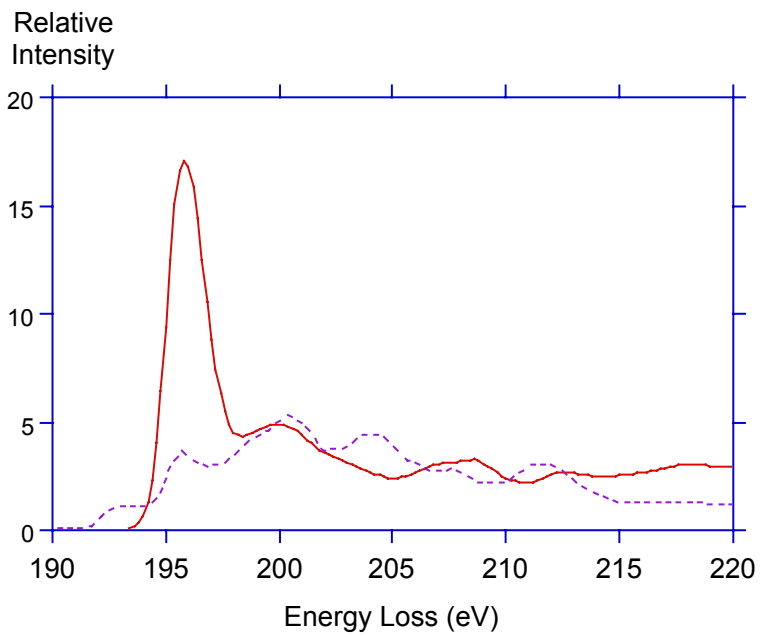

FIG 1 Experimental $\mathrm{B}_{2} \mathrm{O}_{3} \mathrm{~B} \mathrm{~K}$ edge solid line, FEFF8 calculation dashed line

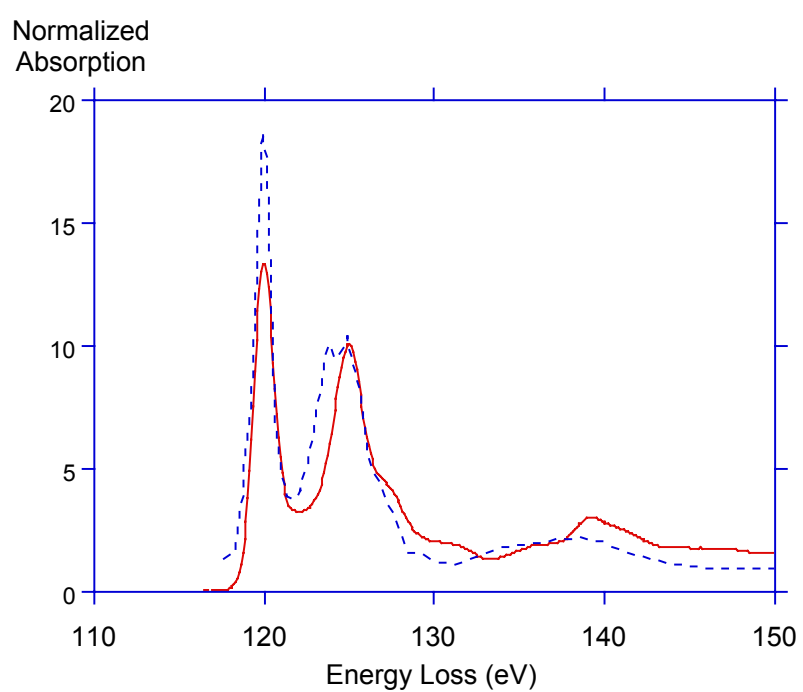

FIG 2 Experimental $\mathrm{BeO} \mathrm{Be} \mathrm{K}$ solid line, Fano resonance calculation dashed line

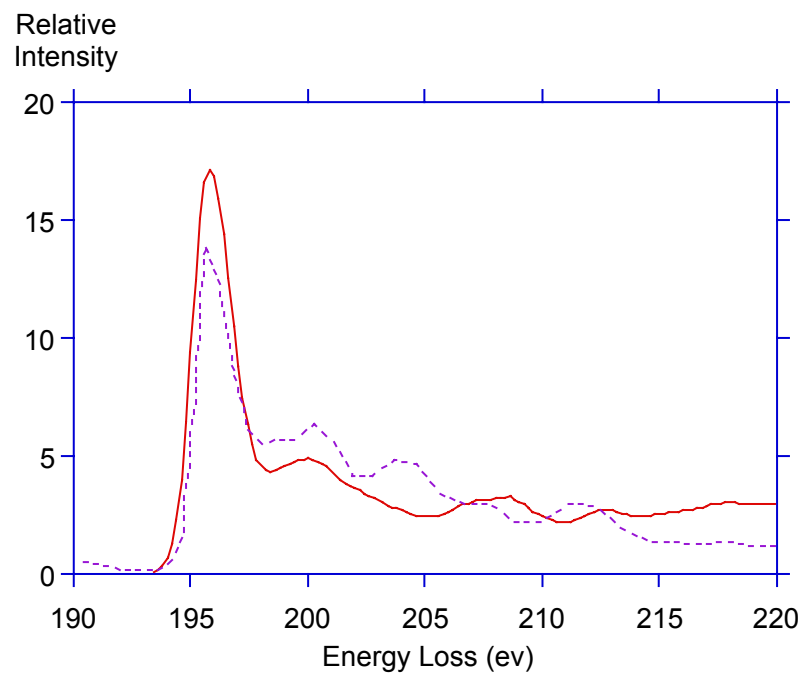

FIG 3 Experimetal $\mathrm{B} \mathrm{K}$ in $\mathrm{B}_{2} \mathrm{O}_{3}$ solid line, Fano resonance calculation dashed line. 
https://doi.org/10.1017/S1431927603444140 Published online by Cambridge University Press 